\title{
A Case Report of Fulminant Type 1 Diabetes Mellitus Caused by Drug Reaction with Eosinophilia and Systemic Symptoms (DRESS) Complicated by Iodine-Induced Thyrotoxicosis
}

\author{
Jingjing Wang $\cdot$ Xuemin Gao $\cdot$ Yuanjie Li $\cdot$ Fan Ping (D
}

Received: February 19, 2019 / Published online: April 1, 2019

(C) The Author(s) 2019

\begin{abstract}
A 46-year-old woman presented with rash, fever, lymphadenopathy, eosinophilia and liver dysfunction. She had been treated with ornidazole for facial rosacea 2 months previously. Two weeks following presentation, she developed diabetic ketoacidosis with exhausted beta islets $(2 \mathrm{~h}$ postprandial laboratory values: glucose $22.0 \mathrm{mmol} / \mathrm{L}$, C-peptide $<0.017 \mathrm{nmol} / \mathrm{L}$, proinsulin $<0.23 \mathrm{pmol} / \mathrm{L}$ ) and negative autoantibodies. Several days thereafter, after having
\end{abstract}

Enhanced Digital Features To view enhanced digital features for this article go to https://doi.org/10.6084/ m9.figshare.7873286

Electronic supplementary material The online version of this article (https://doi.org/10.1007/s13300019-0607-5) contains supplementary material, which is available to authorized users.

J. Wang · F. Ping $(\bowtie)$

Department of Endocrinology, Key Laboratory of Endocrinology of National Health and Family Planning Commission, Peking Union Medical College Hospital, Chinese Academy of Medical Sciences and Peking Union Medical College, Beijing, China

e-mail: pingfan@pumch.cn

X. Gao · Y. Li

Department of General Internal Medicine, Peking Union Medical College Hospital, Chinese Academy of Medical Sciences and Peking Union Medical College, Beijing, China undergone an enhanced computed tomography examination to screen for malignancy, the patient developed thyrotoxicosis with a low iodine absorption rate and super-high urine and serum levels of iodine. The patient was ultimately diagnosed with drug reaction with eosinophilia and systemic symptoms (DRESS), a hypersensitivity reaction to drugs. Studies have shown that immune dysregulation during DRESS renders patients susceptible to new diseases. Here we report a patient who recovered from DRESS but developed fulminant type 1 diabetes complicated with iodine-induced thyrotoxicosis. This case report sheds light on the pathogenesis of DRESS and its sequelae and illustrates that patients with DRESS require longterm care and follow-up.

Keywords: DRESS; Fulminant type 1 diabetes; Iodine-induced thyrotoxicosis

\section{INTRODUCTION}

Drug reaction with eosinophilia and systemic symptoms (DRESS) is a hypersensitivity reaction characterized by fever, rash, eosinophilia, lymphadenopathy and internal organ involvement. It has various clinical presentations, and the affected organs may be hepatic, renal, pulmonary, cardiac, muscular and/or pancreatic [1]. An increasing number of reports have 
documented the manifestation of new diseases in patients who have recovered from DRESS due to immune dysregulation [2]. Fulminant type 1 diabetes (FT1D) is characterized by abrupt onset, profound beta islet destruction requiring insulin replacement and the absence of autoimmune type 1 diabetes-related antibodies; consequently, it is classified as idiopathic type $1 \mathrm{~B}$ diabetes [3]. In a retrospective study which recruited 145 patients with DRESS, five patients subsequently developed FT1D [2]. Onuma et al. reported a series of 15 cases of DRESS complicated with FT1D [4]. Thyrotoxicosis caused by excess iodine uptake is termed as iodine-induced thyrotoxicosis (IIT). The incidence of IIT is low in euthyroid people due to the Wolff-Chaikoff effect [5], while patients with thyroid abnormalities, including Graves' disease, nodular goiter and Hashimoto's disease, are susceptible to IIT [5]. The development of IIT in patients who have recovered from DRESS has never been reported.

\section{CASE REPORT}

A 46-year-old woman presented to our hospital complaining of fever $\left(T_{\max } 39.7^{\circ} \mathrm{C}\right)$ and fatigue. Physical examination revealed enlarged cervical and inguinal lymph nodes. She was treated with antibiotics (cefuroxime and cefoperazone), a nonsteroidal anti-inflammatory drug (nimesulide) and steroid (dexamethasone), however 8 days later macular and non-pruritic rashes had progressed from the trunk to the extremities (Fig. 1). Laboratory tests showed eosinophilia

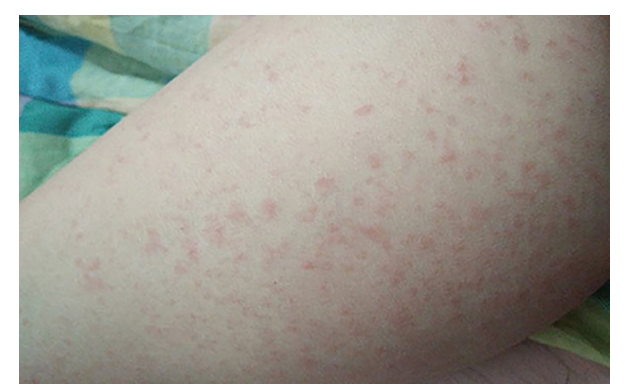

Fig. 1 The rash developed 8 days after the development of fever and progressed from the patient's trunk to the extremities. The rash was macular and non-pruritic $\left(1.25 \times 10^{9} / \mathrm{L}\right)$, liver dysfunction (aspartate transaminase $123 \mathrm{U} / \mathrm{L}$, alanine transaminase $232 \mathrm{U} / \mathrm{L}$ ) and elevated inflammatory markers (C-reactive protein $104.8 \mathrm{mg} / \mathrm{L}$, erythrocyte sedimentation rate $34 \mathrm{~mm} / \mathrm{h}$, ferritin $510 \mathrm{ng} /$ $\mathrm{mL})$. A review of her past medical history revealed the treatment of facial rosacea with ornidazole 2 months prior to fever onset.

Two weeks following the progression of the rashes to the extremities, she developed severe nausea and vomiting with polyuria and polydipsia. Laboratory tests showed hyperglycemia $(34.8 \mathrm{mmol} / \mathrm{L})$ and diabetic ketoacidosis (glucose $34.8 \mathrm{mmol} / \mathrm{L}$, positive urine ketone body, serum $\mathrm{pH}$ 7.16). Further studies showed a normal glycated hemoglobin (6.6\%), negative autoimmune type 1 diabetes-related antibodies (ICA, IA2, and GADA) and elevation of pancreatic enzymes (amylase $132 \mathrm{U} / \mathrm{L}$, lipase $1097 \mathrm{U} / \mathrm{L}$ ). Undetectable serum C-peptide and proinsulin ( $2 \mathrm{~h}$ postprandial: glucose $22.0 \mathrm{mmol} / \mathrm{L}$, C-peptide $<0.017 \mathrm{nmol} / \mathrm{L}$, proinsulin $<0.23 \mathrm{pmol} / \mathrm{L}$ ) indicated that the beta islets were totally destructed. According to criteria for the definite diagnosis of FT1D outlines in the 2012 report of the Committee of the Japan Diabetes Society [6], the patient was diagnosed with FT1D and started on insulin replacement therapy. After intensive insulin replacement, her acute abdominal symptoms subsided immediately, within 1 day.

The patient underwent an enhanced computed tomography (CT) examination with iodinated contrast medium $(100 \mathrm{~mL}$ loversol, containing $32 \mathrm{~g}$ organic iodine or $1.6 \mathrm{~g}$ inorganic iodine) 1 week after the manifestation of FT1D, with the aim to evaluate the pancreas. The enhanced CT scan showed a normal pancreas (Electronic Supplementary Material [ESM] Fig. 1). Initially, the aim of the enhanced thoracoabdominal CT was to rule out malignancy, as the patient was admitted to our hospital for fever of undetermined origin (FUO); however, we subsequently specified the etiology for the FUO as DRESS. At the same time, screening for infection and autoimmune diseases also ruled out these two categories of diseases (ESM Table 1). Five days later, the patient complained of perspiration, hand trembling and palpitation. Physical examination revealed an enlarged thyroid without tenderness. Thyrotoxicosis was 
diagnosed on the basis of decreased levels of thyroxine stimulating hormone and increased levels of both free triiodothyronine and free thyroxine; these abnormalities developed after the use of the contrast medium (iodinated contrast medium) as thyroid function was normal before the CT scan (Table 1). The enhanced CT did not show any nodules of the thyroid (ESM Fig. 2), and color Doppler ultrasonography of the thyroid demonstrated a slightly abundant flow signal and also showed no nodules (ESM Fig. 3). A nuclear scan revealed an extremely low uptake of technetium-99m and radioactive iodine. Based on these findings the patient was diagnosed with IIT and treated with beta blocker to suppress sympathetic tone. An illustration of the entire course is shown in Fig. 2.

We followed up the patient 6 months later, at which time her thyroid function had returned to normal (Table 1). We used a commercialized next-generation sequencing analysis protocol (Cambridge Healthtech Institute [CHI], Inc., Needham, MA, USA) to exclude mutations related to monogenic diabetes mellitus (ESM Table 2), which further consolidated

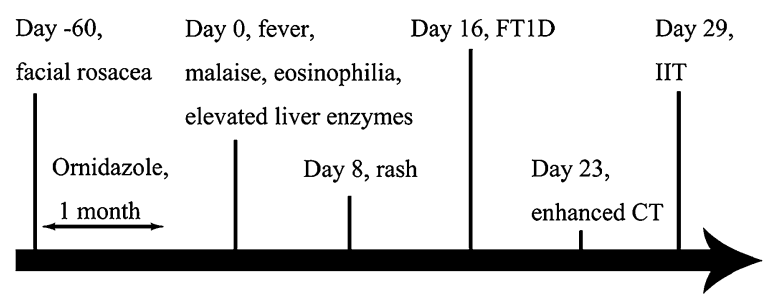

Fig. 2 Flowchart of entire disease course. A 46-year-old woman presented with fever, malaise, lymphadenopathy, eosinophilia and liver dysfunction on day 0 . She had used ornidazole for facial rosacea 2 months previously. On day 8 after presentation, rashes occurred and progressed from the patient's trunk to the extremities. On day 16 after presentation, she developed fulminant type 1 diabetes (FTID). On day 23 after presentation, she underwent an enhanced computed tomography $(C T)$ examination with iodinated contrast media. On day 29 after presentation, she developed iodine-induced thyrotoxicosis (IIT)

the diagnosis of FT1D. We also tested the human leukocyte antigen (HLA) subtypes (ESM Table 3).

All procedures performed in studies involving human participants were in accordance with the ethical standards of the Institutional

Table 1 Laboratory studies on the thyroid function of the patient

\begin{tabular}{llll}
\hline Laboratory parameters & Before application of loversol & After application of loversol & $\mathbf{6}$ months later \\
\hline TSH $(\mu \mathrm{IU} / \mathrm{mL})$ & 0.916 & $0.03 \downarrow$ & 2.398 \\
FT3 $(\mathrm{pg} / \mathrm{mL})$ & 2.65 & $6.83 \uparrow$ & 3.18 \\
FT4 $(\mathrm{ng} / \mathrm{dL})$ & 1.756 & $3.371 \uparrow$ & 1.255 \\
$\mathrm{~T} 3(\mathrm{ng} / \mathrm{mL})$ & - & 1.022 \\
$\mathrm{~T} 4(\mathrm{ug} / \mathrm{mL})$ & 1.044 & - & 7.35 \\
A-TPO $(\mathrm{IU} / \mathrm{mL})$ & 10.37 & $262.9 \uparrow$ & $190.9 \uparrow$ \\
A-Tg $(\mathrm{IU} / \mathrm{mL})$ & $223.2 \uparrow$ & $656.3 \uparrow$ & $519.6 \uparrow$ \\
$\mathrm{TRAb}(\mathrm{IU} / \mathrm{L})$ & $690.6 \uparrow$ & 1.36 & - \\
Serum iodide $(40-90 \mu \mathrm{g} / \mathrm{L})$ & - & $191 \uparrow$ & - \\
$\mathrm{UI} / \mathrm{U}-\mathrm{Cr}(70-860 \mu \mathrm{g} / \mathrm{g} \mathrm{Cr})$ & - & $35,067 \uparrow$ & -
\end{tabular}

TSH Thyroid stimulating hormone, FT3 free triiodothyronine, FT4 free thyroxine, T3 triiodothyronine, T4 thyroxine, $A$-TPO anti-thyroid peroxidase antibodies, $A$-Tg anti-thyroglobulin antibodies, TRAb TSH receptor antibodies, UI urine iodine, $U$ - $C r$ urine creatinine

$\uparrow$, Above the upper limit; $\downarrow$, below the lower limit; -, parameter was not examined 
Review Board of Peking Union Medical College Hospital and with the 1964 Helsinki declaration and its later amendments or comparable ethical standards. Informed consent was obtained from all individual participants included in the study. Additional informed consent was obtained from all individual participants for whom identifying information is included in this article.

\section{DISCUSSION}

Typical DRESS patients would have been exposed to a high-risk medication [7]. A retrospective analysis of 45 patients with DRESS showed that the most common causative drug group was antibiotics (28.9\%), followed by anticonvulsants (26.7\%) [8]. Our patient was exposed to the antibiotic ornidazole, which is not a conventional antibiotic; however, several case reports do show that it is related to fixed drug eruptions [9]. Two other nitro-imidazole drugs, tinidazole and metronidazole, have also been reported to be causes of DRESS [10, 11]. According to the scoring system for classifying DRESS [12], our patient had a score of 5, indicating that she probably suffered from DRESS. In another retrospective study, about $12 \%$ of patients with DRESS had not been exposed to a recognized high-risk drug [12].

In our case, the patient developed FT1D 2 weeks after DRESS flare-up. Her serum level of proinsulin and C-peptide were both undetectable soon after FT1D onset. A recent study revealed that in immune-mediated type 1 diabetes of long duration, the ability of the pancreas to secrete proinsulin persists even at an undetectable serum C-peptide level [13]. Thus, beta islet destruction in FT1D is more severe than that in immune-mediated type 1 diabetes.

In a retrospective study which recruited 145 patients with DRESS, five patients subsequently developed FT1D [2]. An increasing body of data have revealed that defective $\mathrm{T}$ regulatory (Treg) cells play a central role in FT1D [14, 15]. Supporting evidence for the role of Treg cells comes from one study which showed that the FT1D patients in the study had lymphocytic infiltration in both the endocrine and exocrine pancreas, with $98 \%$ of them manifesting elevated levels of serum pancreatic enzymes [6], as the case of our patient (amylase $132 \mathrm{U} / \mathrm{L}$, lipase $1097 \mathrm{U} / \mathrm{L})$.

Treg cell dysfunction signified the resolution stage of DRESS. Treg cells are vigorously activated and expanded in the acute stage of DRESS, while they are functionally defective in the resolution stage [2]. This functional defectiveness may be responsible for the subsequent unopposed inflammatory insult to a specific organ, such as FT1D.

Other theories proposed for DRESS and FT1D include viral reactivation and HLA susceptibility [16]. Yoneda et al. performed immunohistochemistry of the autopsied pancreas of a patient who developed FT1D 3 weeks after DRESS; they detected human cytomegalovirus (HCMV)-positive cells together with macrophages and CD4+/CD8+ T lymphocytes in islets and exocrine areas, indicating that HCMV infection was associated with destruction of the beta cells [17]. As in our case the higher ratio of CD8+ $\mathrm{DR}+/ \mathrm{CD} 8$ and CD8+ CD38+/CD8+ suggested viral infection (Table 2). Onuma et al reviewed 15 cases of DRESS complicated with FT1D reported in the literature and found a high frequency of HLA B62 in those patients [4]. However, our patient did not have the HLA B62 subtype (ESM Table 2). Thus, more data are needed to study the relationship between HLA subtype and DRESS complicated with FT1D.

The low free triiodothyronine/free thyroxine (FT3/FT4) ratio and thyroid iodine absorption rate suggested inflammatory damage to the thyroid and its destruction induced by iodine overload, possibly due to leakage of thyroxine out of the destroyed follicular cells of the thyroid; this mechanism would be consistent with that of IIT [18]. The patient's anti-TPO and anti$\mathrm{Tg}$ antibodies were positive, indicating that she had Hashimoto's disease, which is one of the risk factors for IIT. More importantly, DRESS itself disrupted the immune system and rendered the patient vulnerable to IIT, which further elucidates that immune dysregulation plays a central role in both IIT and FT1D.

The case described herein is the first in which IIT is reported as a sequela of DRESS. Physicians should be aware that as IIT could be a sequela of 
Table 2 Lymphocyte subset

\begin{tabular}{lll}
\hline Parameters & $\begin{array}{l}\text { Onset of FT1D } \\
\text { or } 2 \text { weeks } \\
\text { after the onset } \\
\text { of DRESS }\end{array}$ & $\begin{array}{l}\mathbf{6} \text { months } \\
\text { later }\end{array}$ \\
\hline White blood cell $(/ \mu \mathrm{l})$ & 5600 & 5910 \\
Lymphocyte $(/ \mu \mathrm{l})$ & $1551 \downarrow$ & $1732 \downarrow$ \\
$\mathrm{CD} 19(/ \mu \mathrm{l})$ & $31 \downarrow$ & $76 \downarrow$ \\
$\mathrm{CD} 4 \mathrm{~T}$ cell $(/ \mu \mathrm{l})$ & $540 \downarrow$ & 596 \\
$\mathrm{CD} 8 \mathrm{~T}$ cell $(/ \mu \mathrm{l})$ & 624 & 636 \\
$\mathrm{CD} 4 / \mathrm{CD} 8(/ \mu \mathrm{l})$ & $0.87 \downarrow$ & $0.86 \downarrow$ \\
$\mathrm{CD} 4+\mathrm{CD} 28+/ \mathrm{CD} 4+$ & 96.5 & 96.4 \\
$\mathrm{CD} 8+\mathrm{CD} 28+/ \mathrm{CD} 8+$ & 50.5 & 53.8 \\
$\mathrm{CD} 8+\mathrm{DR}+/ \mathrm{D} 8+$ & $61.3 \uparrow$ & $25.8 \uparrow$ \\
$\mathrm{CD} 8+\mathrm{CD} 38+/ \mathrm{CD} 8+$ & $81.1 \uparrow$ & 48.5 \\
$\mathrm{CD} 16+(\%)$ & 19.4 & 20.9 \\
\hline
\end{tabular}

FTID Fulminant type 1 diabetes, DRESS drug reaction with eosinophilia and systemic symptoms, $C D$ complement (cluster of differentiation)

$\uparrow$, Above the upper limit; $\downarrow$, below the lower limit

DRESS, iodinated substances are inappropriate for the treatment of these patients.

\section{ACKNOWLEDGEMENTS}

We thank all authors for their significant contributions. Jingjing Wang and Fan Ping analyzed the data and wrote the paper, and Yuanjie $\mathrm{Li}$ and Xuemin Gao analyzed the data. We thank the participant of the study for her active cooperation.

Funding. No funding or sponsorship was received for this study or publication of this article.

Authorship. All named authors meet the International Committee of Medical Journal Editors (ICMJE) criteria for authorship for this article, take responsibility for the integrity of the work as a whole, and have given their approval for this version to be published.

Disclosures. Jingjing Wang, Xuemin Gao, Yuanjie Li and Fan Ping have nothing to declare.

Compliance with Ethics Guidelines. All procedures performed in studies involving human participants were in accordance with the ethical standards of the Institutional Review Board of Peking Union Medical College Hospital and with the 1964 Helsinki declaration and its later amendments or comparable ethical standards. Informed consent was obtained from all individual participants included in the study. Additional informed consent was obtained from all individual participants for whom identifying information is included in this article.

Data Availability. All data generated or analyzed during this study are included in this published article/as supplementary information files.

Open Access. This article is distributed under the terms of the Creative Commons Attribution-NonCommercial 4.0 International License (http://creativecommons.org/licenses/ by-nc/4.0/), which permits any noncommercial use, distribution, and reproduction in any medium, provided you give appropriate credit to the original author(s) and the source, provide a link to the Creative Commons license, and indicate if changes were made.

\section{REFERENCES}

1. Chen YC, Chiu HC, Chu CY. Drug reaction with eosinophilia and systemic symptoms: a retrospective study of 60 cases. Arch Dermatol. 2010;146(12):1373-9.

2. Kano Y, Tohyama M, Aihara M, et al. Sequelae in 145 patients with drug-induced hypersensitivity syndrome/drug reaction with eosinophilia and systemic symptoms: survey conducted by the Asian Research Committee on Severe Cutaneous Adverse Reactions (ASCAR). J Dermatol. 2015;42(3):276-82. 
3. American Diabetes Association. Diagnosis and classification of diabetes mellitus. Diabetes Care. 2014;37[Suppl 1]:S81-90.

4. Onuma H, Tohyama M, Imagawa A, et al. High frequency of HLA B62 in fulminant type 1 diabetes with the drug-induced hypersensitivity syndrome. J Clin Endocrinol Metab. 2012;97(12):E2277-E2281281.

5. Leung AM, Braverman LE. Consequences of excess iodine. Nat Rev Endocrinol. 2014;10(3):136-42.

6. Imagawa A, Hanafusa T, Awata T, et al. Report of the Committee of the Japan Diabetes Society on the research of fulminant and acute-onset type 1 diabetes mellitus: new diagnostic criteria of fulminant type 1 diabetes mellitus. J Diabetes Investig. 2012;3(6):536-9.

7. Adwan MH. Drug reaction with eosinophilia and systemic symptoms (DRESS) syndrome and the rheumatologist. Curr Rheumatol Rep. 2017;19(1):3.

8. Nama YH, Parka MR, Namb HJ, et al. Drug reaction with eosinophilia and systemic symptoms syndrome is not uncommon and shows better clinical outcome than generally recognised. Allergol Immunopathol. 2015;43(1):19-24.

9. Emre S, Ahsen H, Aktas A. Ornidazole-induced fixed drug reaction on sole: case report and review of the literature. Cutan Ocul Toxicol. 2017;36(3):294-6.

10. Spriet S, Banks TA. Drug reaction with eosinophilia and systemic symptoms syndrome. Allergy Asthma Proc. 2015;36(6):501-5.

11. Singbal SS, Rataboli PV. Anaphylaxis and hypersensitivity syndrome reactions in increasing severity following repeated exposure to tinidazole. J Postgrad Med. 2005;51(3):243-4.
12. Kardaun SH, Sidoroff A, Valeyrie-Allanore L, et al. Variability in the clinical pattern of cutaneous sideeffects of drugs with systemic symptoms: does a DRESS syndrome really exist? $\mathrm{Br} \mathrm{J}$ Dermatol. 2007;156(3):609-11.

13. Sims E, Bahnson H, Nyalwidhe J, et al. Proinsulin secretion is a persistent feature of type 1 diabetes. Diabetes Care. 2019;42(2):258-64.

14. Haseda F, Imagawa A, Murase-Mishiba $\mathrm{Y}$, Terasaki J, Hanafusa T. CD4(+) CD45RA(-) FoxP3high activated regulatory $\mathrm{T}$ cells are functionally impaired and related to residual insulin-secreting capacity in patients with type 1 diabetes. Clin Exp Immunol. 2013;173(2):207-16.

15. Tada A, Shimada A, Yamada $T$, et al. A mimic of viral double-stranded RNA triggers fulminant type 1 diabetes-like syndrome in regulatory $\mathrm{T}$ cell-deficient autoimmune diabetic mouse. J Immunol. 2011;187(10):4947-53.

16. Yung-Tsu C, Yang C-W, Chu C-Y. Drug reaction with eosinophilia and systemic symptoms (DRESS): an interplay among drugs, viruses, and immune system. Int J Mol Sci. 2017;18(6):1243.

17. Yoneda S, Imagawa A, Fukui K, et al. A histological study of fulminant type 1 diabetes mellitus related to human cytomegalovirus reactivation. J Clin Endocrinol Metab. 2017;102(7):2394-400.

18. Kornelius E, Chiou JY, Yang YS, Peng CH, Lai YR, Huang CN. Iodinated contrast media increased the risk of thyroid dysfunction: a 6-year retrospective cohort study. J Clin Endocrinol Metab. 2015;100(9):3372-9. 